\title{
ON THE HERMITIAN CURVATURE OF SYMPLECTIC MANIFOLDS
}

\author{
LUIGI VEZZONI
}

\begin{abstract}
In this paper we give conditions for the integrability of almost complex structures calibrated by symplectic forms.

We show that in the symplectic case Newlander-Nirenberg theorem reduces to $\nabla^{\prime \prime} N_{J}=0$ and we give integrability conditions in terms of the curvature and the Hermitian curvature of the induced metric.
\end{abstract}

\section{INTRODUCTION}

The interplay between complex and symplectic structures has been recently studied by many authors. Indeed, on any symplectic manifold $(M, \kappa)$ there exists a $\kappa$-calibrated almost complex structure $J$, so that $(M, g, J, \kappa)$ is an almost Kähler manifold.

In the context of almost Kähler geometry it is natural to study the integrability of the almost complex structure.

In 4 Goldberg proved that, if the curvature operator of an almost Kähler manifold $(M, g, J, \kappa)$ commutes with $J$, then $(M, g, J, \kappa)$ is a Kähler manifold.

He also conjectured that an Einstein almost Kähler metric on a compact manifold is a Kähler metric.

If the scalar curvature is nonnegative the conjecture has been proved by Sekigawa in [6].

For a survey, other references and results on this topic we refer to [2].

In this paper we show that, if $(M, g, J, \kappa)$ is an almost Kähler manifold satisfying certain properties, then it is Kähler. Namely we give some conditions on the derivative of the Nijenhuis tensor and on the curvature respectively, in order that $J$ is integrable.

In section 2 we start by recalling some facts and fixing some notations.

In section 3 we prove that if the $(0,1)$-part of the covariant derivative of the Nijenhuis tensor of $J$ vanishes, then $J$ is integrable (theorem 3.3). This result generalizes theorem 2 of 7 ].

In section 4 we consider three types of curvature tensors on $(M, g, J, \kappa)$.

Date: November 2, 2018.

1991 Mathematics Subject Classification. 53C15,53D99, 58A14.

This work was supported by G.N.S.A.G.A. of I.N.d.A.M.. 
Namely, the Riemann curvature $R$, the curvature $\widetilde{R}$ of the Hermitian connection $\widetilde{\nabla}$ and the tensor $R^{J}$ defined by

$$
R^{J}(X, Y, Z, W)=g\left(\nabla_{X} J \nabla_{Y} Z-\nabla_{Y} J \nabla_{X} Z-\nabla_{[X, Y]} J Z, J W\right) .
$$

We show that if $R^{J}$ and $R$ have the same components along certain directions, then $J$ is integrable (theorem 4.2).

Finally we prove that if the bisectional curvature of $g$ and the Hermitian bisectional curvature coincides, then $g$ is a Kähler metric (theorem 4.8).

A key tool in the proof of our results is the existence of generalized normal holomorphic frames (see [7]).

\section{Preliminaries}

Let $M$ be a $2 n$-dimensional (real) manifold.

A symplectic structure on $M$ is a closed non-degenerate 2 -form $\kappa$, i.e. $d \kappa=0$ and $\kappa^{n} \neq 0$. The pair $(M, \kappa)$ is said to be a symplectic manifold.

An almost complex structure on $M$ is a smooth section $J$ of $\operatorname{End}(T M)$, such that $J^{2}=-I d$.

An almost complex structure $J$ is said to be integrable if the Nijenhuis tensor

$$
N_{J}(X, Y)=[J X, J Y]-J[J X, Y]-J[X, J Y]-[X, Y]
$$

vanishes. In view of the celebrated Newlander-Nirenberg theorem, $J$ is integrable if and only if it is induced by a holomorphic structure.

An almost complex structure $J$ on $M$ induces a natural splitting of the complexified of the tangent bundle. Indeed let $T_{J}^{1,0} M, T_{J}^{0,1} M$, be the eigenspaces relatively to $i$ and $-i$ respectively; then $T M \otimes \mathbb{C}=$ $T_{J}^{1,0} M \oplus T_{J}^{0,1} M$. The sections of $T_{J}^{1,0} M, T_{J}^{0,1} M$ are called vector fields of type $(1,0)$ and $(0,1)$ respectively.

We have that $\overline{T_{J}^{1,0} M}=T_{J}^{0,1} M$ and that the map $X \mapsto X-i J X$ defines an isomorphism between $T M$ and $T_{J}^{1,0} M$.

An almost complex structure $J$ is said to be $\kappa$-calibrated if

$$
g[x](\cdot, \cdot)=\kappa[x]\left(\cdot, J_{x} \cdot\right)
$$

is a Hermitian metric on $M$. In this case the triple $(g, J, \kappa)$ is said to be an almost Kähler structure on $M$ and $(M, g, J, \kappa)$ an almost Kähler manifold . Let us denote by $\mathcal{C}_{\kappa}(M)$ the set of the almost complex structure on $M$ calibrated by $\kappa$. It is known that $\mathcal{C}_{\kappa}(M)$ is a non-empty and contractible set (see e.g. [1]). Therefore any symplectic manifold admits almost Kähler structures.

Let $(M, \kappa)$ be a symplectic manifold and $J \in \mathcal{C}_{\kappa}(M)$ : if $J$ is integrable the triple $(g, J, \kappa)$ is said to be a Kähler structure on $M$ and $(M, g, J, \kappa)$ a Kähler manifold (see e.g. [5]). 


\section{Some InTEGRABILITy CONDITIONS}

In this section we give conditions on the covariant derivative of the $\mathrm{Ni}$ jenhuis tensor in order that the complex structure is integrable.

Let $(M, \kappa, J, \kappa)$ be a $2 n$-dimensional almost Kähler manifold. We denote by $\nabla$ the Levi-Civita connection relatively to $g$ and by $R$ the curvature tensor of $g$.

We have the following (see [3] and [7])

Theorem 3.1 (Generalized normal holomorphic frames). For any point $o \in M$ there exists a local complex $(1,0)$-frame, $\left\{Z_{1}, \ldots, Z_{n}\right\}$ around $o$, satisfying the following conditions:

1. $\nabla_{k} Z_{\bar{i}}(o)=0,1 \leq k, i \leq n ;$

2. $\nabla_{k} Z_{i}(o)$ is of type $(0,1), 1 \leq k, i \leq n$;

3. if $G_{r \bar{s}}:=g\left(Z_{r}, Z_{\bar{s}}\right)$, then: $G_{r \bar{s}}(o)=\delta_{r s}, d G_{r \bar{s}}[o]=0$;

4. $\nabla_{r} \nabla_{\bar{k}} Z_{i}(o)=0,1 \leq r, k, i \leq n$, where $\bar{Z}_{i}:=Z_{\bar{i}}$ and $\nabla_{Z_{i}} Z_{j}:=\nabla_{i} Z_{j}$.

By definition $\left\{Z_{1}, \ldots, Z_{n}\right\}$ is said a generalized normal holomorphic frame around $o$.

We recall that the following fundamental relation holds:

$$
2 g\left(\left(\nabla_{X} J\right) Y, Z\right)=g\left(N_{J}(Y, Z), J X\right) .
$$

From the complex extension of (1) it can be easily proved the following (see 7])

Corollary 3.2. The covariant derivative along $(0,1)$-vector fields of the almost complex structure $J$ is a tensor of complex type $(0,1)$, i.e.

$$
\left(\nabla_{Z_{\bar{i}}} J\right) Z_{j}=0
$$

for any $Z_{i}, Z_{j}$ complex vector fields of type $(1,0)$.

Denote by $\nabla^{\prime \prime}$ the $(0,1)$-part of the covariant derivative associated to the Levi-Civita connection, i.e.

$$
\nabla_{W}^{\prime \prime} X=\nabla_{W^{0,1}} X
$$

where $W^{0,1}$ denotes the natural projection of $W$ on $T_{J}^{0,1} M$. As a first application of the generalized normal holomorphic frames we have the following

Theorem 3.3. Assume that

$$
\nabla^{\prime \prime} N_{J}=0
$$

then $(M, g, J, \kappa)$ is a Kähler manifold. 
Proof. Let $o$ be an arbitrary point in $M$ and let $\left\{Z_{1}, \ldots, Z_{n}\right\}$ be a generalized normal holomorphic frame around $o$.

Since $N_{J}\left(T_{J}^{1,0} M \times T_{J}^{1,0} M\right) \subset T_{J}^{0,1} M$ we have

$$
Z_{\bar{i}} g\left(N_{J}\left(Z_{i}, Z_{k}\right), Z_{\bar{k}}\right)=0 .
$$

Therefore, by the assumption $\nabla^{\prime \prime} N_{J}=0$, we get

$$
g\left(N_{J}\left(Z_{i}, Z_{k}\right), \nabla_{\bar{i}} Z_{\bar{k}}\right)=0
$$

and consequently

$$
g\left(N_{J}\left(Z_{i}, Z_{k}\right),\left[Z_{\bar{i}}, Z_{\bar{k}}\right]\right)=0 .
$$

Moreover a direct computation gives

$$
N_{J}\left(Z_{i}, Z_{k}\right)(o)=-\frac{1}{4}\left[Z_{i}, Z_{k}\right](o) .
$$

Hence (2) and (3) imply $N_{J}\left(Z_{i}, Z_{k}\right)(o)=0$, i.e. $J$ is integrable, so that $(M, g, J, \kappa)$ is a Kähler manifold.

Remark 3.4. Let $B$ the $(2,1)$-tensor on $(M, g, J, \kappa)$ defined by

$$
B(X, Y)=J\left(\nabla_{X} J\right) Y-\left(\nabla_{J X} J\right) Y .
$$

Then the Nijenhuis tensor of $J$ is the antisymmetric part of $B$.

In [7] (theorem 2) it is proved that an almost complex structure $J$ is integrable if and only if $\nabla^{\prime \prime} B=0$. Therefore theorem 3.2 generalizes theorem 2 of [7].

Remark 3.5. With respect to a generalized normal holomorphic frame, $\left\{Z_{1}, \ldots, Z_{n}\right\}$, the components of the curvature tensor $R_{i \bar{k} r \bar{s}}(o)$ and $R_{i \bar{k} \overline{r s}}(o)$ are given by

- $R_{i \bar{k} r \bar{s}}(o)=-g\left(\nabla_{\bar{k}} \nabla_{i} Z_{r}, Z_{\bar{s}}\right)(o)$,

- $R_{i \bar{k} \overline{r s}}(o)=g\left(\nabla_{i} \nabla_{\bar{k}} Z_{\bar{r}}, Z_{\bar{s}}\right)(o)$.

In order to study the integrability of a $\kappa$-calibrated almost complex structure $J$ it is useful to introduce the following tensor

$$
L(X, Y, Z, W)=g\left(\left(\nabla_{X} B\right)(Y, Z), W\right),
$$

where $B$ is defined by (4).

A first property of the tensor $L$ is given by the following

Lemma 3.6. This identities hold

$$
\begin{aligned}
& L_{\bar{i} j r \bar{s}}=L_{\overline{j \bar{i}} r} ; \\
& L_{i \bar{j} r s}=L_{i j \bar{r} s}=L_{i j r \bar{s}}=0,
\end{aligned}
$$

for $1 \leq i, j, r, s \leq n$. 
Proof. Let $o$ be an arbitrary point in $M$ and let $\left\{Z_{1}, \ldots, Z_{n}\right\}$ be a generalized normal holomorphic frame around $o$. By the definition of $L$ and the properties of generalized normal holomorphic frames, we get

$$
\begin{aligned}
L_{\bar{i} j r \bar{s}}(o) & =g\left(\left(\nabla_{\bar{i}} B\right)\left(Z_{j}, Z_{r}\right), Z_{\bar{s}}\right)(o) \\
& =g\left(\nabla_{\bar{i}}\left(B\left(Z_{j}, Z_{r}\right)\right), Z_{\bar{s}}\right)(o) \\
& =g\left(\nabla_{\bar{i}}\left(J \nabla_{j} J\right) Z_{r}, Z_{\bar{s}}\right)(o)-i g\left(\nabla_{\bar{i}}\left(\nabla_{j} J\right) Z_{r}, Z_{\bar{s}}\right)(o) \\
& =2 i g\left(\nabla_{\bar{i}} J \nabla_{j} Z_{r}, Z_{\bar{s}}\right)(o)+2 g\left(\nabla_{\bar{i}} \nabla_{j} Z_{r}, Z_{\bar{s}}\right)(o) \\
& =2 i g\left(\nabla_{\bar{i}} J \nabla_{j} Z_{r}, Z_{\bar{s}}\right)(o)+2 R_{\bar{i} j r \bar{s}}(o),
\end{aligned}
$$

i.e.

$$
L_{\bar{i} j r \bar{s}}(o)=2 i g\left(\nabla_{\bar{i}} J \nabla_{j} Z_{r}, Z_{\bar{s}}\right)(o)+2 R_{\bar{i} j r \bar{s}}(o) .
$$

By a direct computation we get

$$
\begin{aligned}
L_{\bar{i} j r \bar{s}}(o)= & 2 i Z_{\bar{i}} g\left(J \nabla_{j} Z_{r}, \bar{Z}_{s}\right)(o)+2 i g\left(\nabla_{j} Z_{r}, J \nabla_{\bar{i}} Z_{\bar{s}}\right)(o) \\
& +2 R_{\bar{i} j r \bar{s}}(o) .
\end{aligned}
$$

Then we have

$$
\begin{aligned}
L_{\bar{i} j r \bar{s}}(o)= & 2 i Z_{\bar{i}} g\left(J \nabla_{j} Z_{r}, Z_{\bar{s}}\right)(o)+2 i Z_{j} g\left(Z_{r}, J \nabla_{\bar{i}} Z_{\bar{s}}\right)(o) \\
& -2 i g\left(Z_{r}, \nabla_{j} J \nabla_{\bar{i}} Z_{\bar{s}}\right)(o)+2 R_{\bar{i} j r \bar{s}}(o) \\
= & -2 Z_{\bar{i}} g\left(\nabla_{j} Z_{r}, Z_{\bar{s}}\right)(o)+2 Z_{j} g\left(Z_{r}, \nabla_{\bar{i}} Z_{\bar{s}}\right)(o) \\
& -2 i g\left(Z_{r}, \nabla_{j} J \nabla_{\bar{i}} Z_{\bar{s}}\right)(o)+2 R_{\bar{i} j r \bar{s}}(o) \\
= & -2 g\left(\nabla_{\bar{i}} \nabla_{j} Z_{r}, Z_{\bar{s}}\right)(o)+2 g\left(Z_{r}, \nabla_{j} \nabla_{\bar{i}} Z_{\bar{s}}\right)(o) \\
& -2 i g\left(Z_{r}, \nabla_{j} J \nabla_{\bar{i}} Z_{\bar{s}}\right)(o)+2 R_{j \bar{i} \bar{s} r}(o) \\
= & -2 R_{\bar{i} j r \bar{s}}(o)+2 R_{\bar{j} \bar{s} r}(o) \\
& -2 i g\left(Z_{r}, \nabla_{j} J \nabla_{\bar{i}} Z_{\bar{s}}\right)(o)+2 R_{\overline{j i} \bar{s} r}(o) \\
= & -2 i g\left(Z_{r}, \nabla_{j} J \nabla_{\bar{i}} Z_{\bar{s}}\right)(o)+2 R_{\bar{j} \bar{i} \bar{s} r}(o)
\end{aligned}
$$

In a similar way we obtain

$$
L_{j \bar{i} \bar{s} r}(o)=-2 i g\left(Z_{r}, \nabla_{j} J \nabla_{\bar{i}} Z_{\bar{s}}\right)(o)+2 R_{j \bar{i} \bar{s} r}(o) .
$$

Therefore

$$
L_{\bar{i} j r \bar{s}}(o)=L_{j \bar{i} \bar{s} r}(o) .
$$

The second part of the proof is straightforward.

As a corollary of theorem 3.3 we have the following (see also [7]):

Proposition 3.7. Assume that

$$
L\left(Z_{\bar{i}}, Z_{i}, Z_{j}, Z_{\bar{j}}\right)=0,
$$

for any complex vector fields $Z_{i}, Z_{j}$ of type $(1,0)$; then $(M, g, J, \kappa)$ is a Kähler manifold. 
Proof. Let $\left\{Z_{1}, \ldots, Z_{n}\right\}$ be a generalized normal holomorphic frame around $o$. A direct computation gives

$$
L\left(Z_{\bar{i}}, Z_{i}, Z_{j}, Z_{\bar{j}}\right)(o)=g\left(\nabla_{i} Z_{j}, \nabla_{\bar{i}} Z_{\bar{j}}\right)(o) ;
$$

hence $\nabla_{i} Z_{j}(o)=0$.

Therefore $N_{J}\left(Z_{i}, Z_{j}\right)(o)=-\frac{1}{4}\left[Z_{i}, Z_{j}\right](o)=0$.

\section{Curvature and integrability}

In this section we give an integrability condition in terms of curvature.

We start defining the following tensor

$$
R^{J}(X, Y, Z, W)=g\left(\nabla_{X} J \nabla_{Y} Z-\nabla_{Y} J \nabla_{X} Z-\nabla_{[X, Y]} J Z, J W\right) .
$$

We have

Lemma 4.1. Let $\left\{Z_{1}, \ldots, Z_{n}\right\}$ be a generalized normal holomorphic frame around $o$, then

$$
R_{\bar{i} j r \bar{s}}^{J}(o)=-i g\left(\nabla_{\bar{i}} J \nabla_{j} Z_{r}, Z_{\bar{s}}\right)(o) .
$$

Proof. By the definition of $R^{J}$ it follows that

$$
\begin{aligned}
R_{\bar{i} j r \bar{s}}^{J}(o)= & -i g\left(\nabla_{\bar{i}} J \nabla_{j} Z_{r}, Z_{\bar{s}}\right)(o)+i g\left(\nabla_{j} J \nabla_{\bar{i}} Z_{r}, Z_{\bar{s}}\right)(o) \\
& -g\left(\nabla_{\left[Z_{\bar{i}}, Z_{j}\right]} Z_{r}, Z_{\bar{s}}\right)(o) \\
= & -i g\left(\nabla_{\bar{i}} J \nabla_{j} Z_{r}, Z_{\bar{s}}\right)(o)+i g\left(\nabla_{j} J \nabla_{\bar{i}} Z_{r}, Z_{\bar{s}}\right)(o) .
\end{aligned}
$$

By corollary 3.2. we have $\left(\nabla_{\bar{i}} J\right) Z_{j}=0$. Therefore we obtain

$$
g\left(\nabla_{j} J \nabla_{\bar{i}} Z_{r}, Z_{\bar{s}}\right)(o)=i g\left(\nabla_{j} \nabla_{\bar{i}} Z_{r}, Z_{\bar{s}}\right)(o)=0
$$

and then

$$
R_{\bar{i} j r \bar{s}}^{J}(o)=-i g\left(\nabla_{\bar{i}} J \nabla_{j} Z_{r}, Z_{\bar{s}}\right)(o) .
$$

The previous lemma and equation (5) give us

$$
L_{\bar{i} j r \bar{s}}(o)=-2 R_{\bar{i} j r \bar{s}}^{J}(o)+2 R_{\bar{i} j r \bar{s}}(o) .
$$

Moreover equation (7) and lemma (3.6) imply the following

Theorem 4.2. If

$$
R^{J}\left(Z_{\bar{i}}, Z_{j}, Z_{i}, Z_{\bar{i}}\right)=R\left(Z_{\bar{i}}, Z_{j}, Z_{i}, Z_{\bar{j}}\right)
$$

for any $Z_{i}, Z_{j}$ of type $(1,0)$, then $J$ is integrable.

Remark 4.3. The hypothesis of the previous theorem can be replaced by

$$
R^{J}(J X, Y, X, J Y)=R(J X, Y, X, J Y)
$$

for any $X, Y$ real vector fields. 
Let $\widetilde{\nabla}$ be the Hermitian connection on $(M, g, J, \kappa) ; \widetilde{\nabla}$ is the connection defined by

$$
\widetilde{\nabla}=\nabla-\frac{1}{2} J \nabla J
$$

Then $\widetilde{\nabla}$ preserves the metric $g$, the almost complex structure $J$ and its torsion is given by the Nijenhuis torsion, namely

$$
\widetilde{\nabla} g=0, \quad \widetilde{\nabla} J=0, \quad T^{\widetilde{\nabla}}=\frac{1}{4} N_{J} .
$$

Let us denote by $\widetilde{R}$ the curvature tensor of $\widetilde{\nabla}$.

From [6] we have the following

Lemma 4.4 ([6]). For any $X, Y, Z, W$ we have the following formula

$$
\begin{aligned}
\widetilde{R}(X, Y, Z, W)= & \frac{1}{2} R(X, Y, Z, W)+\frac{1}{2} R(X, Y, J Z, J W) \\
& -\frac{1}{4} g\left(\left(\nabla_{X} J\right)\left(\nabla_{Y} J\right) Z-\left(\nabla_{Y} J\right)\left(\nabla_{X} J\right) Z, W\right) .
\end{aligned}
$$

Now we have

Lemma 4.5. Let $\left\{Z_{1}, \ldots, Z_{n}\right\}$ be a local $(1,0)$-frame. Then

$$
\widetilde{R}_{i \bar{j} r \bar{s}}=R_{i \bar{j} r \bar{s}}+\frac{1}{4} L_{i \bar{j} r \bar{s}}
$$

for any $1 \leq i, j, r, s \leq n$.

Proof. Let $\left\{Z_{1}, \ldots, Z_{n}\right\}$ be a local $(1,0)$-frame; from lemma 4.4 we have

$$
\begin{aligned}
\widetilde{R}_{i \bar{j} r \bar{s}} & =\frac{1}{2} R_{i \bar{j} r \bar{s}}+\frac{1}{2} R_{\overline{i j} r \bar{s}}-\frac{1}{4} g\left(\left(\nabla_{i} J\right)\left(\nabla_{\bar{j}} J\right) Z_{r}, Z_{\bar{s}}\right)+\frac{1}{4} g\left(\left(\nabla_{\bar{j}} J\right)\left(\nabla_{i} J\right) Z_{r}, Z_{\bar{s})}\right. \\
& =R_{i \bar{j} r \bar{s}}+\frac{1}{4} g\left(\left(\nabla_{\bar{j}} J\right)\left(\nabla_{i} J\right) Z_{r}, Z_{\bar{s}}\right) .
\end{aligned}
$$

From corollary 3.2 we have $\left(\nabla_{\bar{j}} J\right) Z_{r}$; then we get

$$
g\left(\left(\nabla_{i} J\right)\left(\nabla_{\bar{j}} J\right) Z_{r}, Z_{\bar{s}}\right)=0 .
$$

Therefore

$$
\begin{aligned}
\widetilde{R}_{i \bar{j} r \bar{s}}= & R_{i \bar{j} r \bar{s}}+i \frac{1}{4} g\left(\left(\nabla_{\bar{j}} J\right) \nabla_{i} Z_{r}, Z_{\bar{s}}\right)-\frac{1}{4} g\left(\left(\nabla_{\bar{j}} J\right) J \nabla_{i} Z_{r}, Z_{\bar{s}}\right) \\
= & R_{i \bar{j} r \bar{s}}+i \frac{1}{4} g\left(\nabla_{\bar{j}} J \nabla_{i} Z_{r}, Z_{\bar{s}}\right)-i \frac{1}{4} g\left(J \nabla_{\bar{j}} \nabla_{i} Z_{r}, Z_{\bar{s}}\right)+\frac{1}{4} g\left(\left(\nabla_{\bar{j}} \nabla_{i} Z_{r}, Z_{\bar{s}}\right)\right. \\
& +\frac{1}{4} g\left(\left(J \nabla_{\bar{j}} J \nabla_{i} Z_{r}, Z_{\bar{s}}\right)\right. \\
= & R_{i \bar{j} r \bar{s}}+i \frac{1}{4} g\left(\nabla_{\bar{j}} J \nabla_{i} Z_{r}, Z_{\bar{s}}\right)+\frac{1}{4} g\left(\nabla_{\bar{j}} \nabla_{i} Z_{r}, Z_{\bar{s}}\right)+\frac{1}{4} g\left(\left(\nabla_{\bar{j}} \nabla_{i} Z_{r}, Z_{\bar{s}}\right)\right. \\
& +i \frac{1}{4} g\left(\left(\nabla_{\bar{j}} J \nabla_{i} Z_{r}, Z_{\bar{s}}\right)\right. \\
= & R_{i \bar{j} r \bar{s}}+i \frac{1}{2} g\left(\nabla_{\bar{j}} J \nabla_{i} Z_{r}, Z_{\bar{s}}\right)+\frac{1}{2} g\left(\nabla_{\bar{j}} \nabla_{i} Z_{r}, Z_{\bar{s}}\right)
\end{aligned}
$$


i.e.

$$
\widetilde{R}_{i \bar{j} r \bar{s}}=R_{i \bar{j} r \bar{s}}+i \frac{1}{2} g\left(\nabla_{\bar{j}} J \nabla_{i} Z_{r}, Z_{\bar{s}}\right)+\frac{1}{2} R_{\bar{j} i r \bar{s}} .
$$

Let assume now that $\left\{Z_{1}, \ldots, Z_{n}\right\}$ is a generalized normal holomorphic frame around a point $o$. Then equations (9) and (5) imply

$$
\widetilde{R}_{i \bar{j} r \bar{s}}(o)=R_{i \bar{j} r \bar{s}}(o)+\frac{1}{4} L_{\bar{j} i r \bar{s}}(o) .
$$

Theorem 4.2 and lemma 4.5 imply the following

Theorem 4.6. If

$$
\widetilde{R}\left(Z_{i}, Z_{\bar{i}}, Z_{j}, Z_{\bar{j}}\right)=R\left(Z_{i}, Z_{\bar{i}}, Z_{j}, Z_{\bar{j}}\right)
$$

for any $Z_{i}, Z_{j}(1,0)$-fields, then $(M, g, J, \kappa)$ is a Kähler manifold.

Remark 4.7. The hypothesis of theorem 4.6 can be given as

$$
\widetilde{R}(X, J X, Y, J Y)=R(X, J X, Y, J Y) .
$$

for any pair of real vector fields $X, Y$

The last theorem can be stated in terms of the bisectional curvatures of $\nabla$ and $\widetilde{\nabla}$.

Let $p \in M$ and $v, w$ be two unit vector of $T_{p} M$. Then the holomorphic bisectional curvature of the planes $\sigma_{1}=\langle v, J v\rangle, \sigma_{2}=\langle w, J w\rangle$ is defined by

$$
K[p]\left(\sigma_{1}, \sigma_{2}\right)=R(v, J v, w, J w) .
$$

We denote by $\widetilde{K}[p]\left(\sigma_{1}, \sigma_{2}\right)$ the Hermitian bisectional curvature (i.e. the bisectional curvature of the Hermitian connection). For any $p \in M$ let $\mathcal{P}_{p}^{1,1}(M)$ be the set of $J$-invariant planes in $T_{p} M$.

Hence we get

Theorem 4.8. If

$$
K(p)\left(\sigma_{1}, \sigma_{2}\right)=\widetilde{K}(p)\left(\sigma_{1}, \sigma_{2}\right)
$$

for any $p \in M$ and $\sigma_{1}, \sigma_{2} \in \mathcal{P}_{p}^{1,1}(M)$, then $J$ is an integrable almost complex structure and therefore $(M, g, J, \kappa)$ is a Kähler manifold. 


\section{REFERENCES}

[1] Holomorphic curves in symplectic geometry. Edited by Michéle Audin and Jacques Lafontaine. Progress in Mathematics, 117. Birkhuser Verlag, Basel, 1994. xii+328 pp.

[2] Apostolov V., Draghici T.: The curvature and the integrability of almost-Kähler manifolds: a survey, Symplectic and contact topology: interactions and perspectives (Toronto, ON/Montreal, QC, 2001), 25-53, Fields Inst. Commun., 35, Amer. Math. Soc., Providence, RI, 2003.

[3] P. de Bartolomeis and A. Tomassini, On Formality of Some Symplectic Manifolds, Internat. Math. Res. Notices 2001, no. 24, pp. 1287-1314..

[4] S. I. Goldberg, Integrability of almost-Kähler manifolds, Proc. Amer. Math. Soc. 21 (1969), pp. 96-100.

[5] S. Kobayashi and K. Nomizu, Foundations of differential geometry. Vol. II.Interscience Publishers John Wiley \& Sons, Inc., New York-London-Sydney 1969.

[6] K. Sekigawa, On some Einstein almost Kähler manifolds, J. Math. Soc. Japan Vol.39 (1987), No.4, pp. 677-684.

[7] L.Vezzoni, A generalization of the normal holomorphic frames in Symplectic manifolds. Boll. Un. Mat. Ital. To appear.

Dipartimento di Matematica "L. Tonelli", Università di Pisa, Largo Bruno Pontecorvo 5, 56127 Pisa, Italy

E-mail address: vezzoni@mail.dm.unipi.it 